\title{
Equinococosis: Actualización en Terminologías Utilizadas desde la Biología a la Terapéutica
}

\author{
Echinococcosis: Update on Terminologies Used from Biology to Therapeutics
}

Claudio Rojas ${ }^{1,2}$ \& Carlos Manterola ${ }^{1,3}$

ROJAS, C. \& MANTEROLA, C. Equinococosis: Actualización en terminologías utilizadas desde la biología a la terapéutica. Int. J. Morphol., 39(4):1240-1252, 2021.

RESUMEN: El diagnóstico y tratamiento de las equinococosis requiere de la participación de especialistas multidisciplinarios,por lo que, estandarizar la terminología utilizada es fundamental. Para armonizar la terminología sobre bases científicas y lingüísticas sólidas, la Asociación Mundial de Equinococosis generó un proceso de consenso formal. Los principales logros de este proceso fueron: realizar una actualización de la nomenclatura actual de Echinococcus spp.; generar un acuerdo sobre nombres de enfermedades causadas por Echinococcus spp.: equinococosis quística (EQ), equinococosis alveolar (EA) y equinococosis neotropical (NE), y la exclusión de los demás nombres; ejecutar un acuerdo sobre el uso restringido del adjetivo "hidatido" para referirse al quiste y al líquido debido a $E$. granulosus sensu lato; y un acuerdo sobre una descripción estandarizada de las intervenciones quirúrgicas para EQ, de acuerdo con el marco de "Acceso, Apertura, Resección e Integridad del quiste" (AARI). Además, se enumeraron 95 términos "aprobados" y 60 "rechazados". Las recomendaciones proporcionadas serán aplicables a publicaciones científicas y comunicación con profesionales. El objetivo de este manuscrito fue generar un documento de estudio referente al uso de terminología actualizada en echinococcosis desde la biología a la terapéutica.

PALABRAS CLAVE: Echinococcus; Echinococcosis; Equinococcosis quística; Echinococcosis alveolaris; Echinococcosis neotropical; Terminología.

\section{INTRODUCCIÓN}

Hablar de Echinococcosis o hidatidosis es remontarse a tiempos de Hipócrates (460-379 a.C.) quien describió como hydatis a estructuras con forma de gotas, bolsas o vejiga de agua de forma común para cisticercos y cenuros. Hartmann en 1694 demostró la naturaleza animal de los quistes a quien denominó Echinococcus hydatidosus (racimo de erizos a los protoescolex); y en 1695 observó un ejemplar adulto en el intestino de un perro. En 1782 Ephraim, demostró la naturaleza verminosa del quiste denominándola Taenia visceralis, a la que Batsch (1786) denominó Hydatigena granulosa. Posteriormente, Rudolphi (1801), la incorporó en el género Echinococcus como se conoce hoy: Echinococcus granulosus. Finalmente, los trabajos de Siebold (1852) y de Naunyn (1863), demostraron el ciclo heteroxénico entre el hospedero definitivo (perro) y los intermediarios (animales y humanos) (Cordero del Campillo, 2008; Turnes, 2009).

Al ser la equinococosis una zoonosis cuyas especies tienen ciclos de vida complejos, diversos hospedadores, y un impacto relevante en la salud pública de las sociedades; se requiere de una activa participación de especialistas de múltiples disciplinas (agricultura, ecología, zoología, parasitología, medicina veterinaria, salud pública, subes-pecialidades de la medicina interna, cirugía, y economía entre otras), cada cual con sus propias jergas; pero que deben colaborar con un propósito común que es resolver los múltiples problemas que genera la infección por Echinococcus spp., requiriendo de un vocabulario común, siendo importante estandarizar la terminología utilizada en el campo de la equinococosis (Vuitton et al., 2020).

Las especies de Echinococcus (spp.), son parásitos de la clase Céstoda y pertenecen al filo Platyhelminthes. Son responsables de diversas enfermedades en humanos, principalmente la equinococosis quística (EQ); a pesar que el concepto aparece en publicaciones científicas bajo las denominaciones "hidatidosis","enfermedad hidatídica", "quiste hidatídico", etc.). Del mismo modo, la equinococosis alveolar (EA), aparece como Echinococcus alveolaris, "hidatidosis alveolar", "quiste hidatídico multilocular","hidatidosis multilocular",

\footnotetext{
${ }^{1}$ Programa de Doctorado en Ciencias Médicas, Universidad de La Frontera, Temuco, Chile.

${ }^{2}$ Instituto de Salud Pública. Universidad Austral de Chile, Valdivia, Chile.

${ }^{3}$ Centro de Estudios Morfológicos y Quirúrgicos (CEMyQ), Universidad de La Frontera, Temuco, Chile.
} 
"alveococosis", "hidatidosis multilocular", etc.) (Vuitton et al., 2011; Deplazes et al., 2017). Y, la equinococosis neotropical (EN); aparece como "enfermedad poliquística hidatídica", "equinococosis poliquística", "hidatidosis del Nuevo Mundo", etc.) (Kern et al., 2017), lo que demuestra la falta de estandarización de la terminología utilizada.

Por otra parte, el desarrollo de técnicas de biología molecular y el conocimiento del genoma del E. granulosus (Zheng et al., 2013) y del E. multilocularis (Tsai et al., 2013), ha permitido conocer nuevas especies dentro del género Echinococcus, lo que ha incrementado el conocimiento taxonómico de estos parásitos. Lo mismo ha ocurrido a nivel de proteómica, metabolómica, inmunología y epidemiología (Zhang et al., 2014; Wen et al., 2019); de tal modo que hay 9 especies reconocidas y caracterizadas por las secuencias de sus genomas, y definidas en términos de especies hospedadoras y áreas endémicas (Wen et al.; Manterola et al., 2021), no obstante, en las publicaciones se sigue utilizando la denominación E. granulosus como nombre de una sola especie responsable de la EQ.

El problema se traspasa también a la clínica, lo que se asocia también al hecho que la EQ ha sido definida como enfermedades "huérfanas" o "enfermedades desatendidas" (Booth, 2018); y "Enfermedades Tropicales Desatendidas" (World Health Organization, 2015), aunque la mayoría de los casos no se encuentran en áreas tropicales.

Por otra parte, la Federación Mundial de Parasitólogos (FMP), respaldó la denominada Nomenclatura Estandarizada de Enfermedades Parasitarias (SNOPAD), que data desde 1988; y que estableció reglas para los nombres de las enfermedades parasitarias (Kassai et al., 1988). De este modo, el nombre de las enfermedades deriva del género del parásito con un sufijo en "-osis"; así, Echinococcus da "equinococosis"; aunque para esta enfermedad prevalece la coexistencia de varios nombres, lo que impacta directamente en la búsqueda de información en las bases de datos; y que genera, además, malentendidos entre investigadores y profesionales (Kassai, 2006a,b).

Es importante recordar el rol de la Asociación Internacional de Hidatidología, fundada en Uruguay en 1941; con el objetivo de coordinar el control de la equinococosis, especialmente en los países endémicos de América del Sur. Esta, cambió posteriormente de nombre a Asociación Mundial de Equinococosis (WAE), durante uno de sus congresos (Bucarest, 2015).

En 1985, se creó el Grupo de Trabajo Informal de la OMS sobre Equinococosis (IWGE), con los objetivos de: crear una red de científicos que trabajaran en ciencias bási- cas en el ámbito de la equinococosis; y en la estandarización de prácticas para el diagnóstico y tratamiento de Echinococcus spp. y sus enfermedades asociadas (en línea con las prioridades de la OMS) (Eckert \& Thompson, 2017); lo que se mantiene hasta hoy.

Es así como en una sesión plenaria del $27^{\circ}$ Congreso Mundial de Equinococosis en Argel, Argelia, 2017; se destacó la necesidad de armonizar la terminología de la equinococosis, sobre bases científicas y lingüísticas sólidas, estableciéndose un grupo de trabajo para un consenso de recomendaciones aplicables a las publicaciones científicas.

En 2020, la WAE publicó el producto de una consulta realizada a 49 participantes representando a 15 países (trabajo iniciado en octubre de 2017 y finalizado en febrero de 2020); agrupados enun grupo de estudio de "Especies y epidemiología"; otro, de "Biología e inmunología"; y otro de "Aspectos clínicos" sobre acuerdos de aprobación o rechazo de terminologías utilizadas en echinococcosis (Vuitton et al., 2020).

El objetivo de este manuscrito fue generar un documento de estudio referente al uso de terminología actualizada en echinococcosis desde la biología a la terapéutica.

\section{TERMINOLOGÍAS ACTUALIZADAS}

Desde la perspectiva genética y epidemiológica de las especies, hay una serie de términos recomendados, que se resumen en la Tabla I.

Desde el punto de vista de la biología del parásito, los diversos componentes del huevo de todas las especies Echinococcus spp. y del quiste de EQ, se proporcionan figuras aclaratorias que corrige las definiciones anteriores (Figs. 1 y 2).

La terminología de los aspectos biológicos e inmunológicos de las especies de Echinococcus spp., se resume en la Tabla II. Así mismo, tanto desde el punto de vista de la biología e inmunología, como de la clínica; el término "hidatidosis" debiera abandonarse. Esto, incluye todos los nombres de enfermedades u operaciones compuestas por el radical "hidatido" (por ejemplo, en "hidatidosis" o "hidatidosis", etc.) y, que cuando se utilice el adjetivo "hidatido", no debe utilizarse para calificar entidades diferentes a las debidas a E. granulosus sensu lato, por lo que nunca se debe utilizar para E. multilocularis, E. vogeli y E. oligarthra. Aunque esto resulta controversial mirado desde la perspectiva de la zoología, que utiliza la taxonomía para 
ROJAS, C. \& MANTEROLA, C. Equinococosis: Actualización en terminologías utilizadas desde la biología a la terapéutica. Int. J. Morphol., 39(4):1240-1252, 2021.

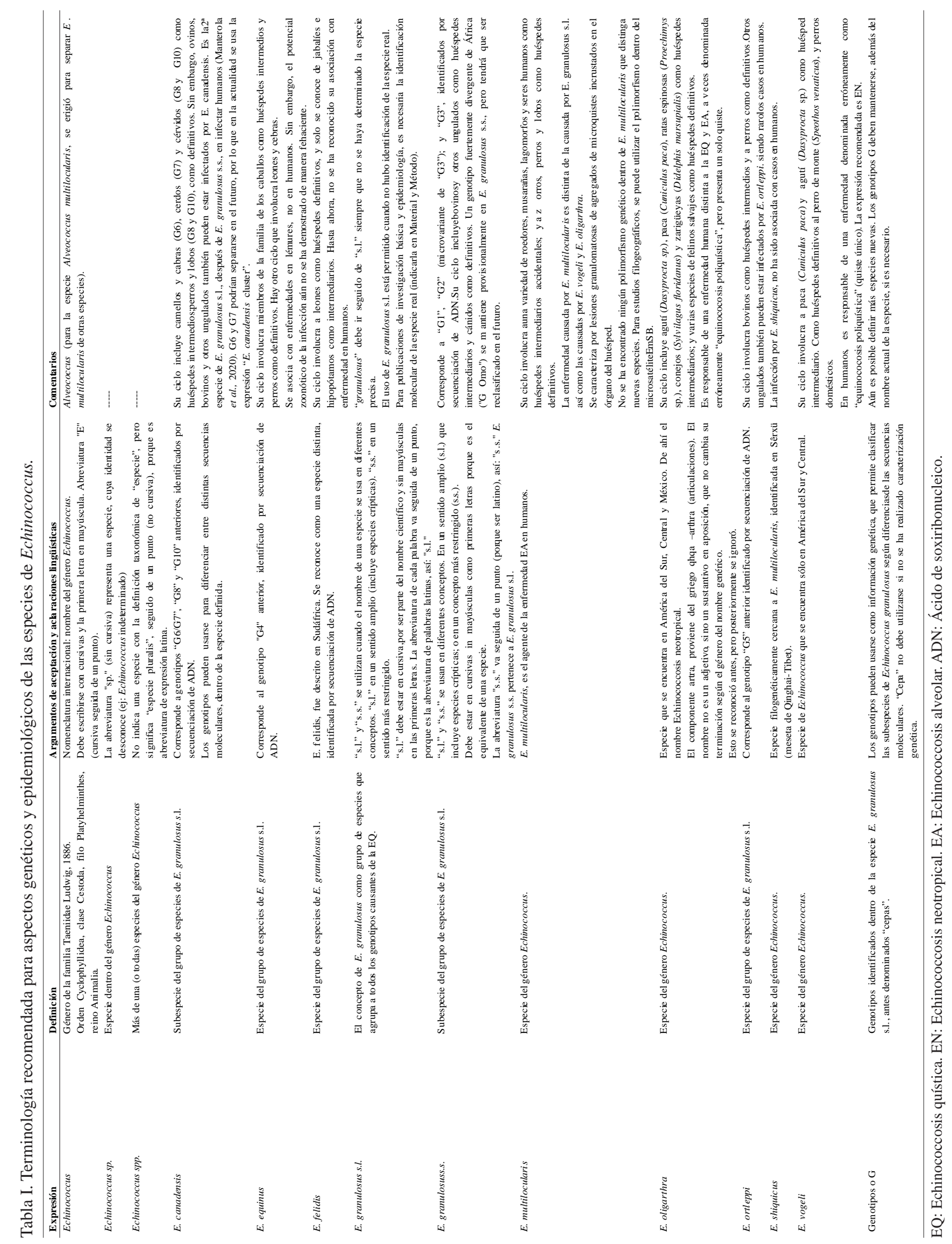


dar orden a la teoría de ascendencia común de Darwin (1809-1882), iniciada con Aristóteles (384-322 a.C.) y consolidada por Linnaeus (1707-1778) (Hickman et al., 2016).

Permite clasificar a los animales e identificarlos de forma binomial en latín (código internacional de nomenclatura zoológica); el primer nombre inicia en mayúscula e identifica el género; y el segundo en minúscula o epíteto específico y exclusivo de la especie, por ejemplo: perro (Canis lupus familiaris), humano (Homo sapiens), Taenia solium, etc. De este modo, el uso de sp. y spp. deben entenderse como, especie del género Echinococcus o Echinococcus sp. en singular y en plural Echinococcus spp.

Por otro lado, las parasitosis humanas son por lo general de ciclo monoxénico o ciclo directo, por lo que el nombre de la enfermedad que causan corresponde al género del parásito independiente de su estado, por ejemplo: giardiasis (trofozoíto o quiste). En las parasitosis de ciclo heteroxénico el estado evolutivo del parásito causante de la enfermedad asigna el hombre; es así como la teniasis es una parasitosis intestinal humana (Taeniasolium y $T$. saginata) y la equinococcosis es una parasitosis intestinal canina. Por su parte sus estados larvarios o metacéstodos en el humano generan cisticercosis (Cysticercus cellulosae) e hidatidosis (Hydatide).

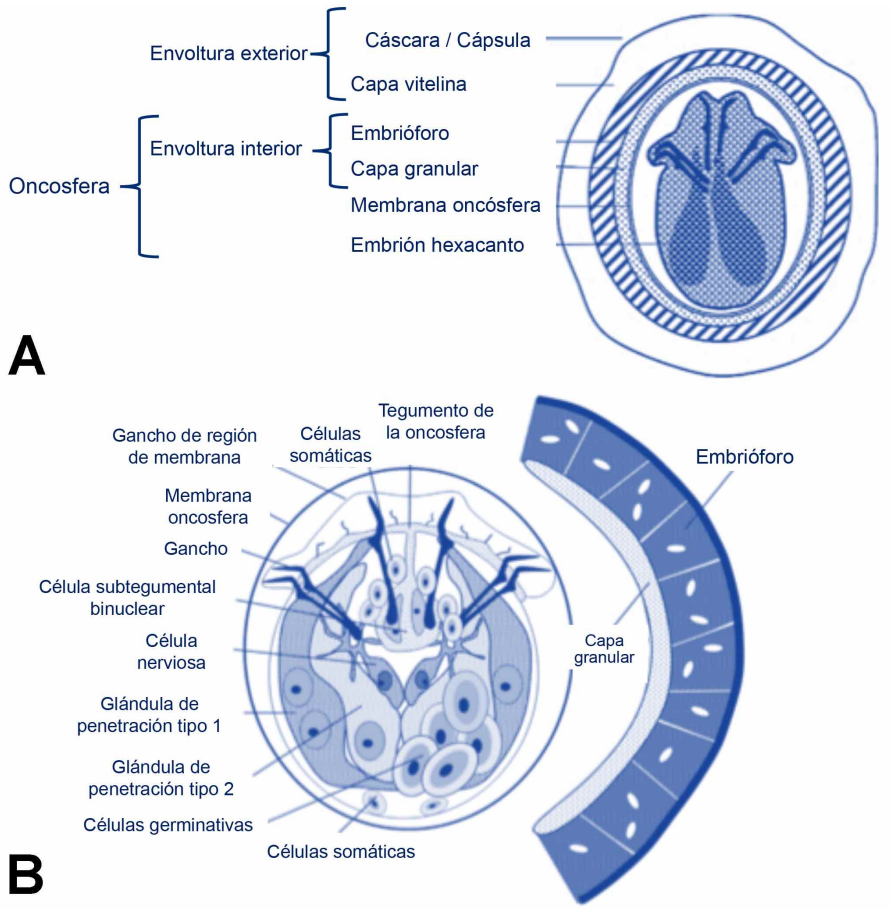

Fig. 1. Representación gráfica del huevo y la oncosfera de Echinococcus spp. A. Estructura y simetría en el patrón de ganchos y organización celular del embrión hexacanto. B. Organización celular de la oncosfera.

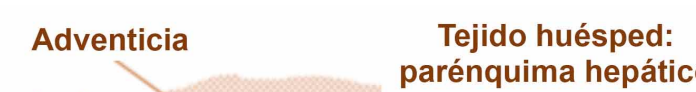

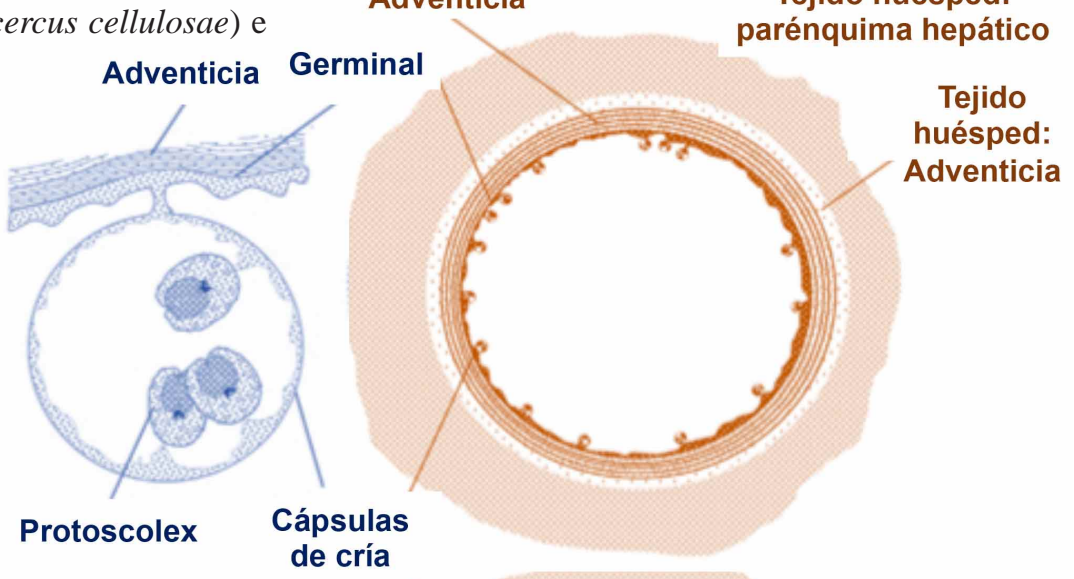

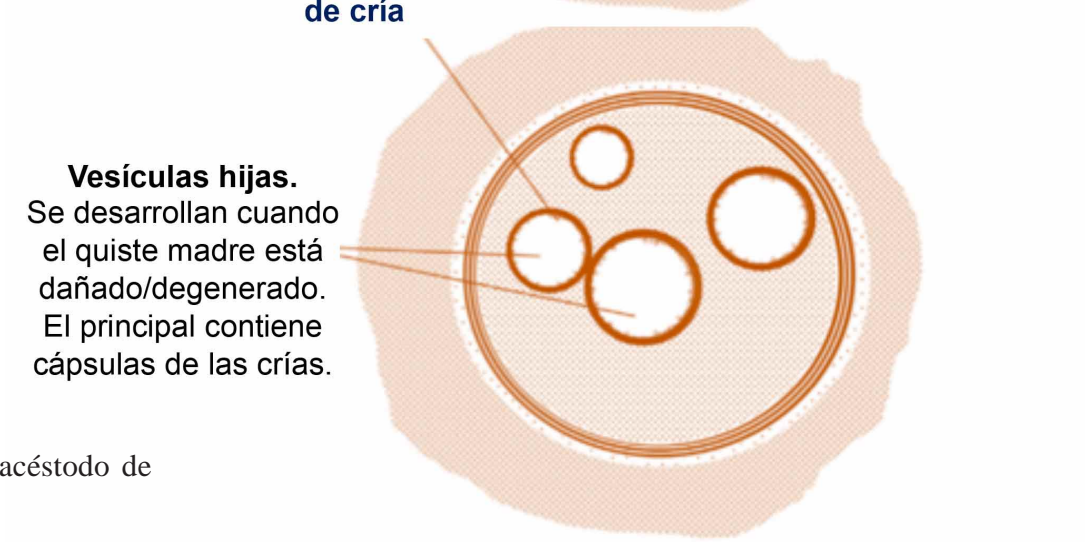


ROJAS, C. \& MANTEROLA, C. Equinococosis: Actualización en terminologías utilizadas desde la biología a la terapéutica. Int. J. Morphol., 39(4):1240-1252, 2021.

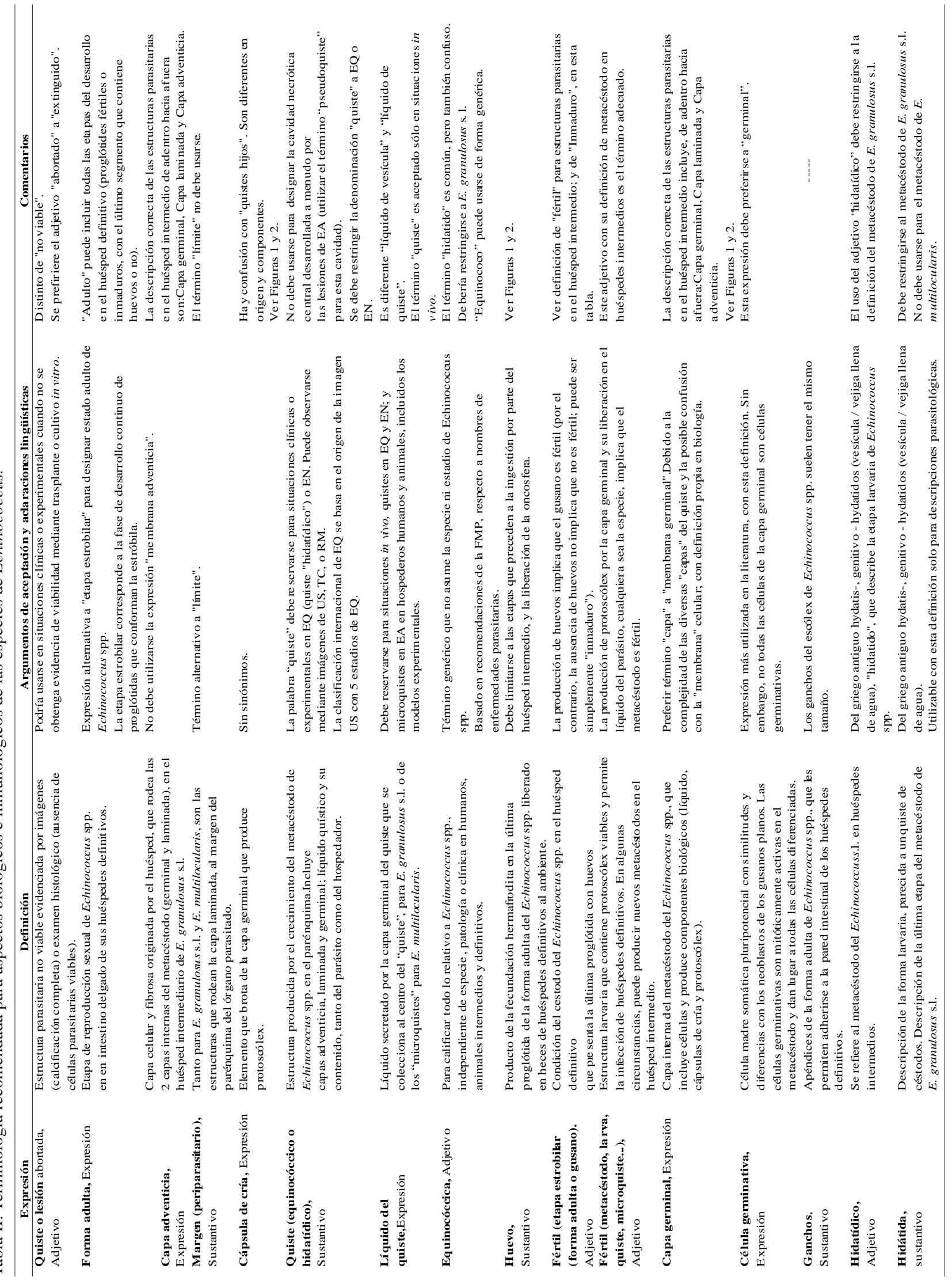


ROJAS, C. \& MANTEROLA, C. Equinococosis: Actualización en terminologías utilizadas desde la biología a la terapéutica. Int. J. Morphol., 39(4):1240-1252, 2021.

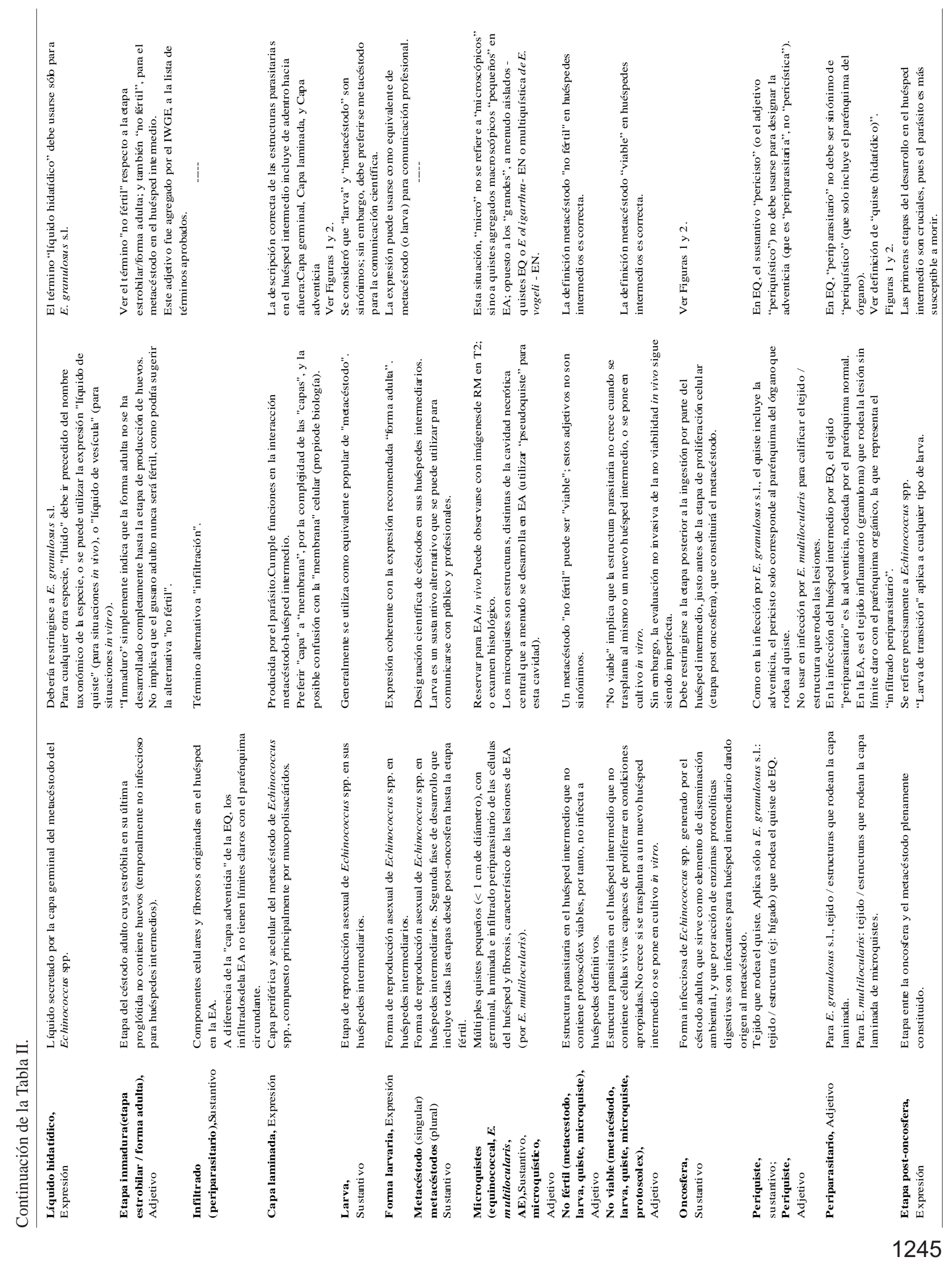




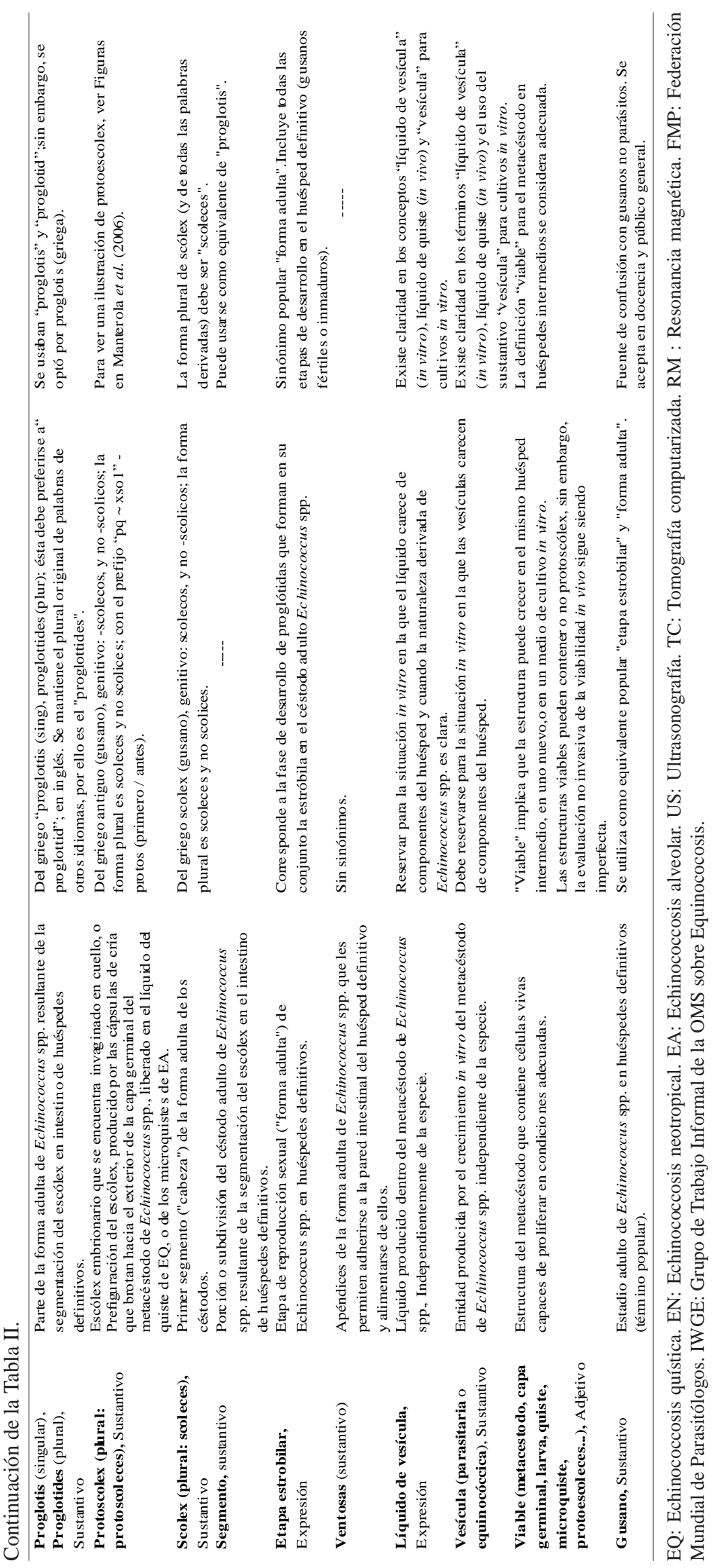

Desde un punto de vista clínico y terapéutico, se sugiere modificar la terminología en algunos aspectos, que se resumen en la Tabla III. Respecto de procedimientos quirúrgicos destinados a la extirpación de quistes de EQ, se presenta una propuesta simple para ser considerada al momento de publicar resultados quirúrgicos. Consta de 4 niveles de calificación: Acceso (laparotomía, laparoscopía, robótica); Apertura del quiste ("quiste no abierto" o NOP, versus "quiste abierto" u OP); tipo de Resección (quistectomía, hepatectomía, o trasplante hepático); e, Integridad o completitud de la resección (total, subtotal y parcial). La descripción del marco unificado AARI (AORC en inglés [Approach, cyst Opening, Resection type, Completeness of resection]), se puede apreciar en la Tabla IV y Figura 3. La descripción precisa del procedimiento realizado debe completar los términos obligatorios (descripción de las capas parasitarias removidas, área en centímetros cuadrados o porcentaje de quiste realmente resecado, cierre de las comunicaciones con las vías biliares, etc.).

Finalmente, hay una serie de términos tanto genéticos, epidemiológicos, biológicos, clínicos y terapéuticos, que se han ido eliminando y que se sugiere no volver a utilizar. 
ROJAS, C. \& MANTEROLA, C. Equinococosis: Actualización en terminologías utilizadas desde la biología a la terapéutica. Int. J. Morphol., 39(4):1240-1252, 2021.

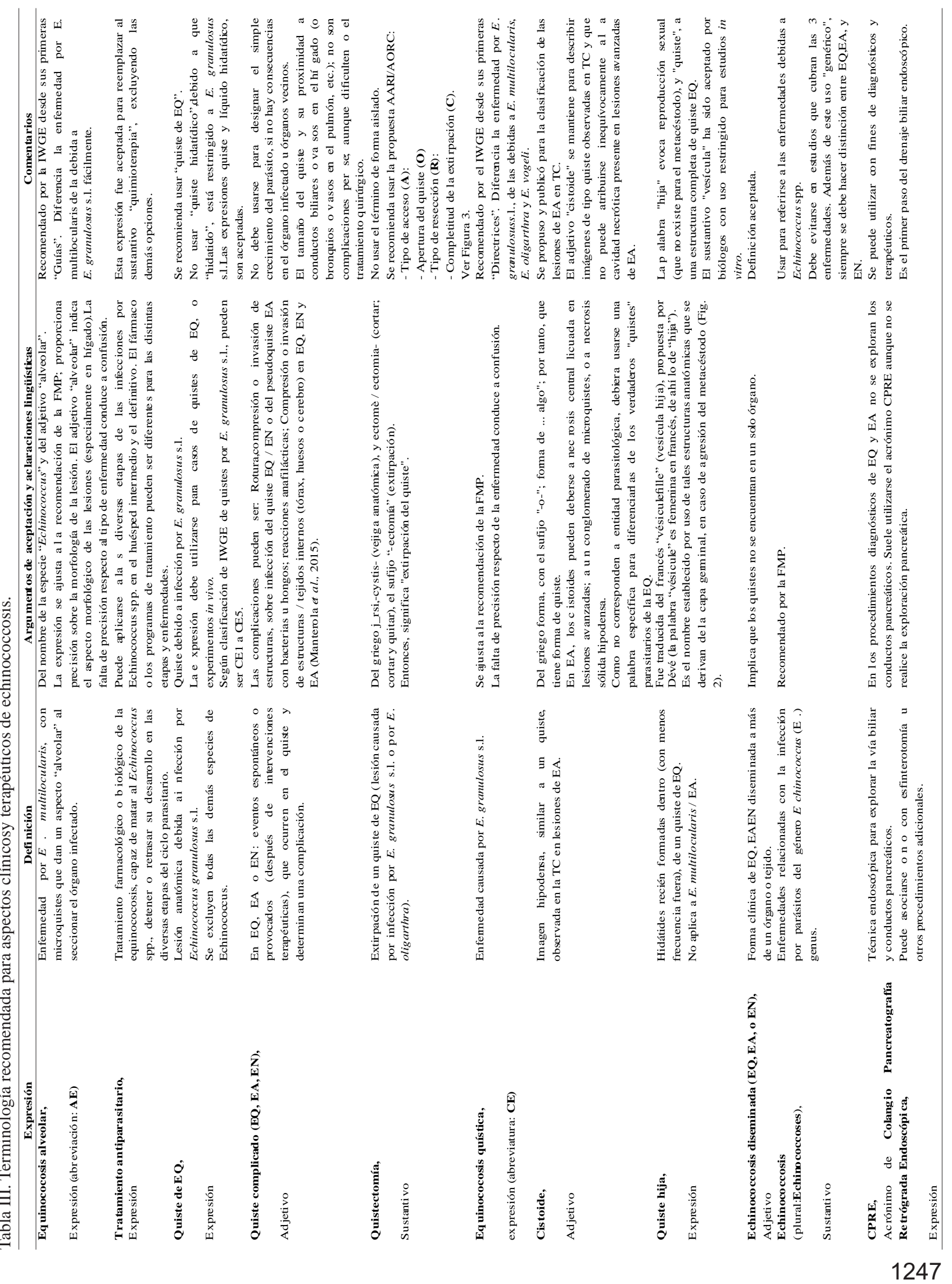


ROJAS, C. \& MANTEROLA, C. Equinococosis: Actualización en terminologías utilizadas desde la biología a la terapéutica. Int. J. Morphol., 39(4):1240-1252, 2021.

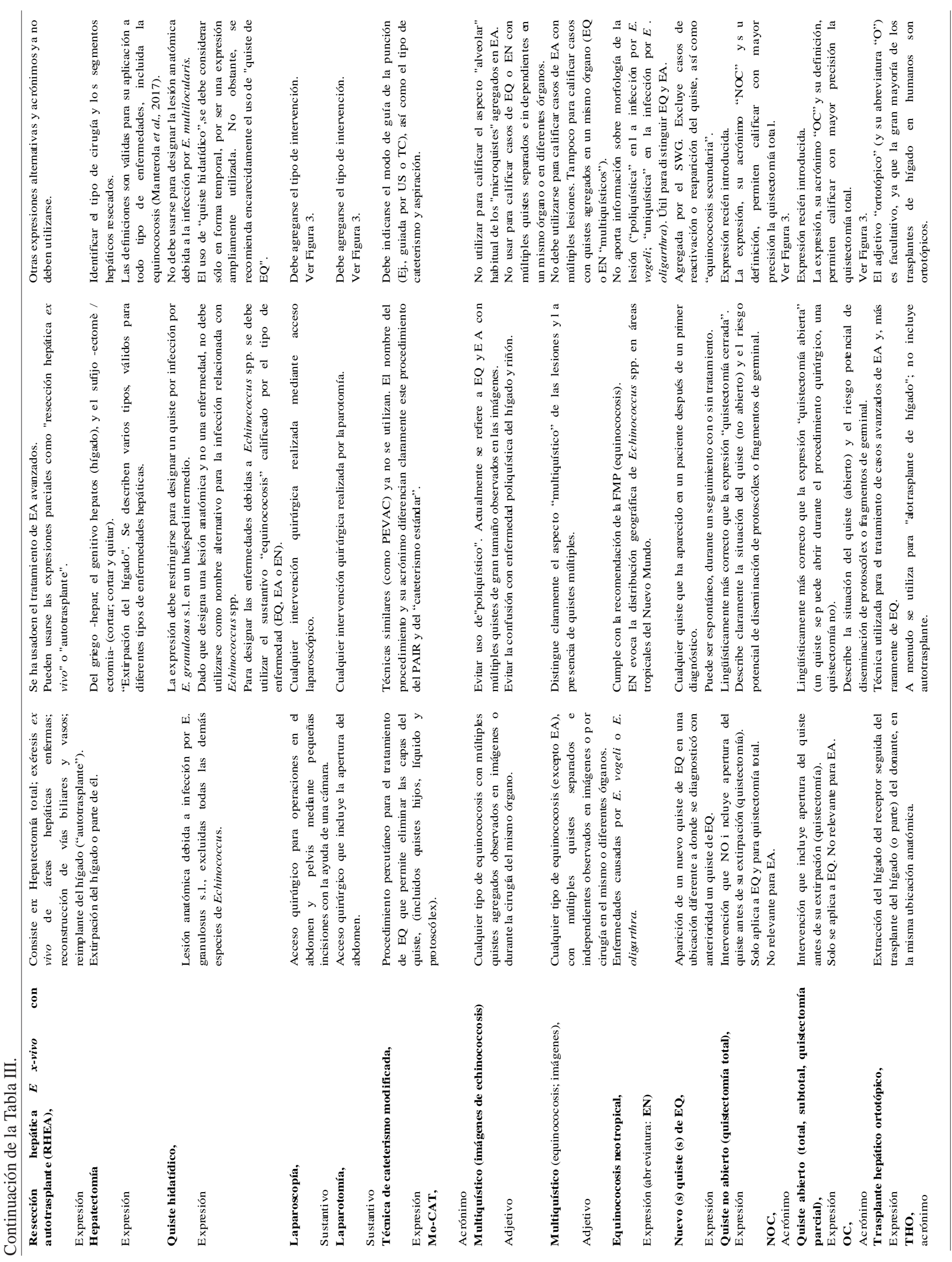


ROJAS, C. \& MANTEROLA, C. Equinococosis: Actualización en terminologías utilizadas desde la biología a la terapéutica. Int. J. Morphol., 39(4):1240-1252, 2021.

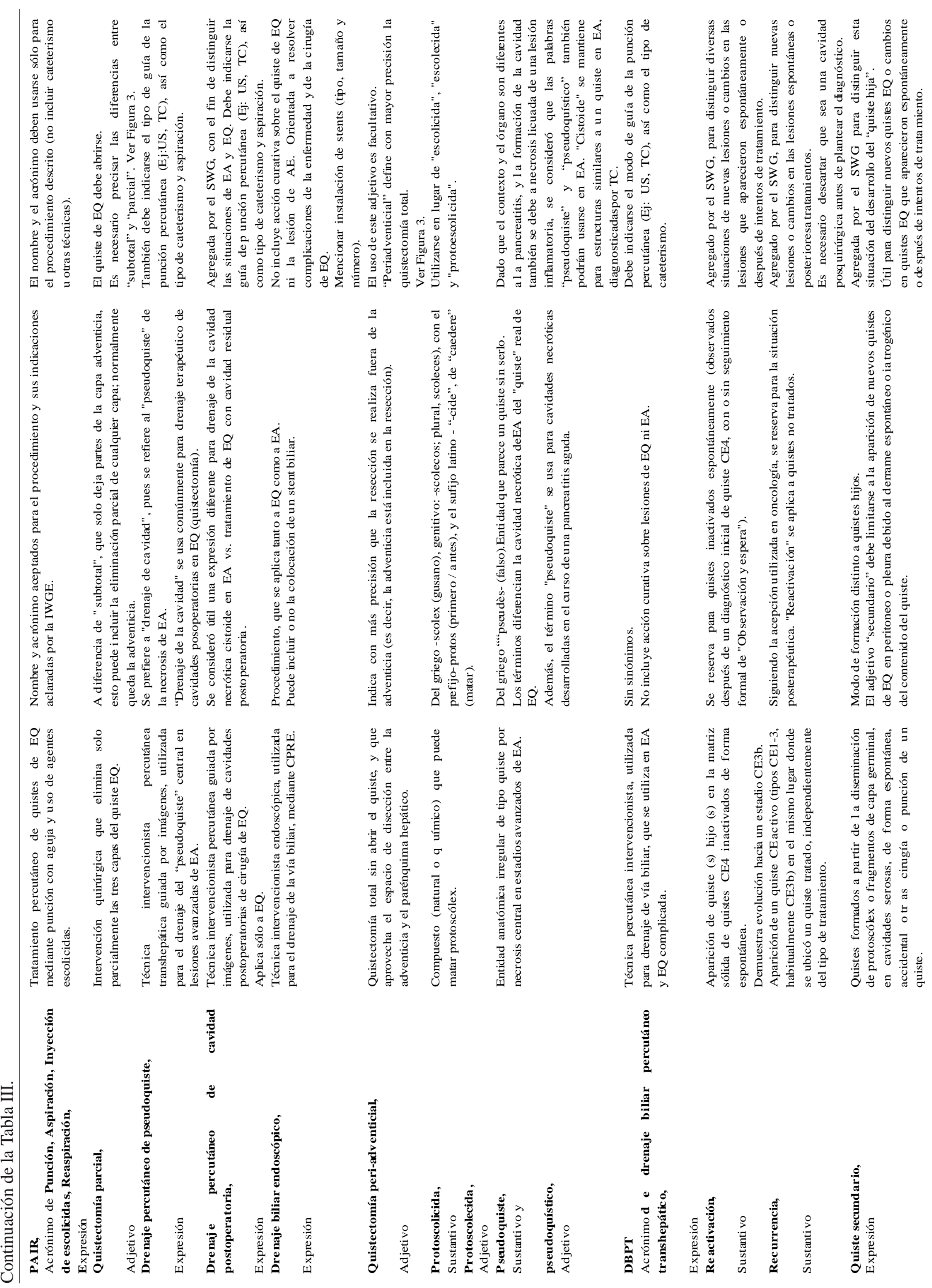




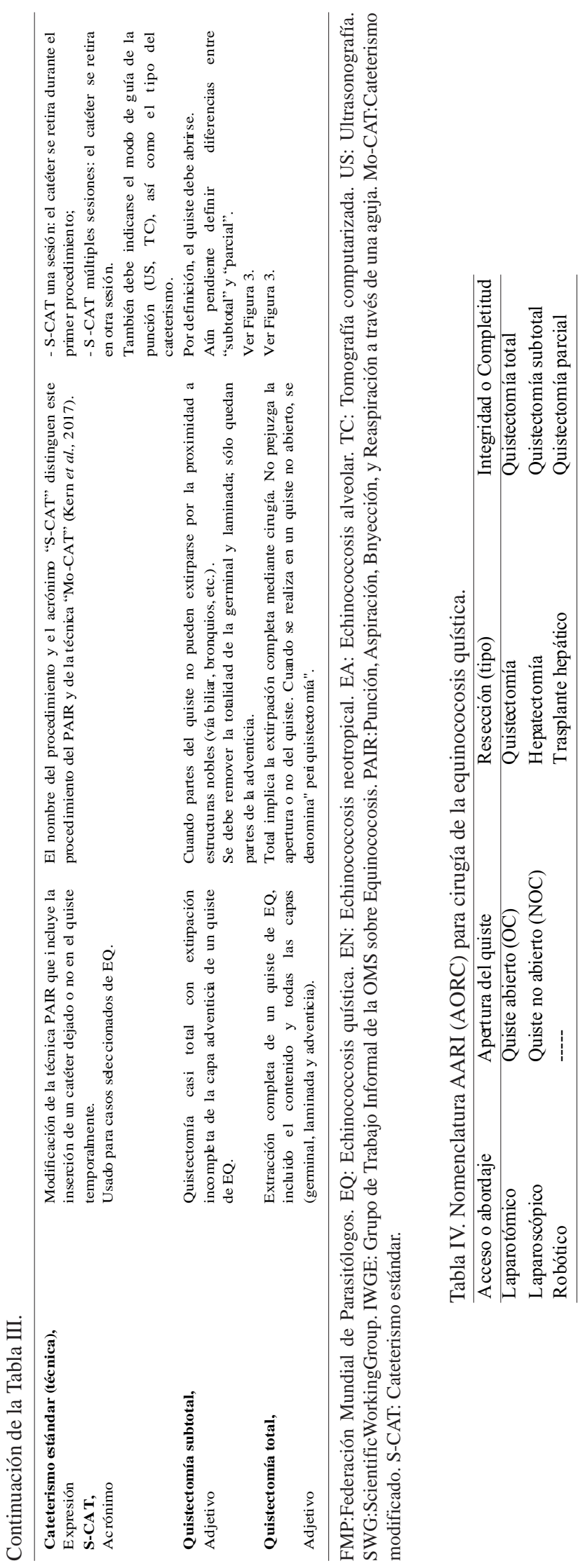

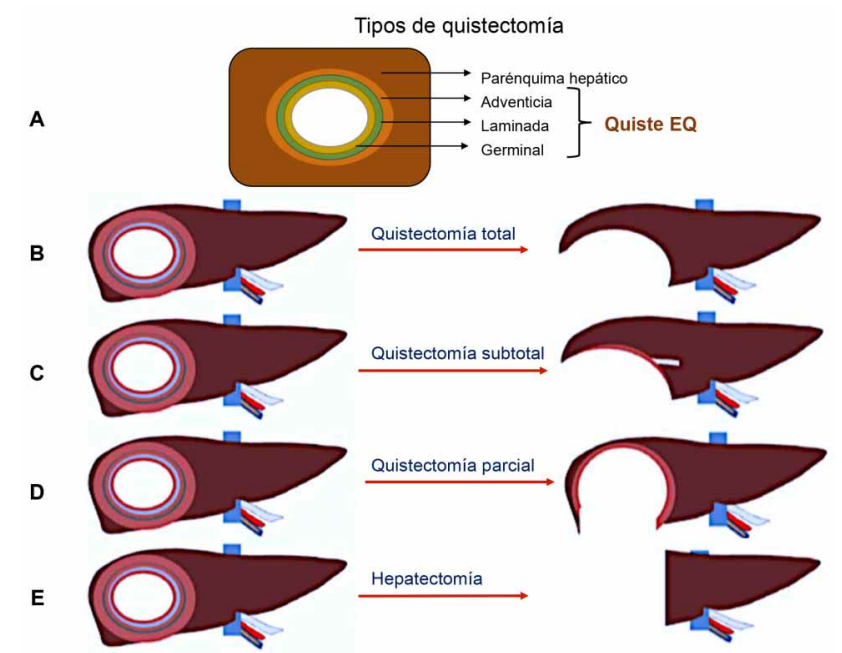

Fig. 3. Nomenclatura AARI ("AORC" en inglés), para la cirugía de la EQ. A. Esquema de la estructura de un quiste hidatídico. Compuesto desde adentro hacia afuera de capas germinal, laminada y adventicia. B. La quistectomía total requiere la resección de las tres capas completamente. C. La quistectomía subtotal requiere la resección casi total de las tres capas; Solo se conservan partes de la capa adventicia debido a la seguridad quirúrgica. D. La quistectomía parcial se refiere a la resección incompleta de cualquiera de las tres capas (generalmente de la adventicia) debido al problemas técnicos y de seguridad. E. La hepatectomía requiere la resección en el bloque delquiste y el parénquima hepático circundante, siguiendo las reglas de una resección hepática.

\section{DISCUSIÓN}

Actualmente, se dispone de una definición genética precisa para 9 especies (McManus, 2013; Nakao et al., 2013): E. granulosus sensu stricto (Manterola et al., 2008; Zheng et al.), E. canadensis (E. Intermedius [G6/7], E. borealis [G8] y E. canadensis [G10]) (Nakao et al.; Manterola et al., 2020, 2021), E. ortleppi (Addy et al., 2017), E. felidis (Manterola et al., 2021), y E. equinus (Macin et al., 2021), dentro de E. granulosus sensu lato (Manterola et al., 2020, $2021)$ y responsable de la equinococosis quística, $E$. multilocularis (Tsai et al., 2013); E. shiquicus, responsable de la equinococosis alveolar (Vuitton et al., 2011; Deplazes et al.); E. vogeli (Bittencourt-Oliveira et al., 2018); y E. oligarthra, responsable de la equinococosis neotropical (Romig et al., 2017) (Tabla I).

Las variaciones en torno al radical "hidatido" se han utilizado durante mucho tiempo para designar enfermedades debidas a Echinococcus spp. La presencia de quistes "hidatídicos" ha sido reconocida durante mucho tiempo como característica de esta enfermedad parasitaria (Vuitton et al., 2011; Eckert \& Thompson). La especie fue denominada como Taenia echinococcus antes de convertirse en $E$. 
granulosus. Al no ser una teniasis en humanos, prevaleció el concepto de "enfermedad hidatídica", "hidatidosis" o "quiste hidatídico". Así, la hidatidosis que corresponde al nombre coherente de la enfermedad; sobre una contradicción con las normas SNOPAD y se inicia la adopción del nombre echinococcosis, termino que actualmente es usado en consenso.

Posteriormente, se identificó como una especie distinta de E. multilocularis, causante de la Echinococcosis alveolaris (Tsai et al.; Eckert \& Thompson). Por todo ello, es que el término "hidatidos" se ha considerado fuente de confusión tanto epidemiológica como clínica, pues se ha utilizado el mismo prefijo para distintas enfermedades (Kern et al., 2017; Vuitton et al., 2020), por lo que no se ha de utilizar términos basados en la raíz "hidatídico" para designar enfermedad debida a Echinococcus spp.; y, restringir el uso de "hidatídico" sólo para calificar una lesión debida a $E$. granulosus s.l. en huéspedes intermedios.

En relación a la lesión quística, debería preferirse la denominación "quiste de EQ" a la de "quiste hidatídico".

En referencia a la infección por E. oligarthra (no oligarthrus) y E. vogeli, y a las enfermedades que pueden causar en humanos; se decidió utilizar el concepto "neotropical", debido a que ambas son infecciones de las áreas tropicales del Nuevo Mundo, quedando como "Infección por Equinococosis Neotropical”. Esto, entre otras cosas, permite reducir la confusión con las enfermedades poliquística hepática y renal, y autosómica dominante, caracterizada por la coexistencia de quistes múltiples en hígado y riñón (en ocasiones con predilección sólo por uno de los dos órganos) (Cornec-Le Gall et al., 2018).

El debate entre "quiste hija" y "vesícula hija", se dirimió por "quiste hija", debido a su amplia utilización para designar los quistes nuevos desarrollados en peritoneo y pleura después de la rotura del quiste "madre".

Las anteriores propuestas aprobadas por la IWGE, es decir, la clasificación "EQ" de los quistes de EQ (WHO Informal Working Group, 2003); y para lesiones de Echinococcosis alveolaris (Kern et al., 2006), se mantienen.

Desde el punto de vista terapéutico, se considera el sistema de descripción de intervenciones quirúrgicas en pacientes con EQ, denominado AARI (Acceso, Apertura del quiste, tipo de resección, Integridad de la resección; AORC en inglés); que propone entre otras, la eliminación del concepto periquistectomía y su reemplazo por quistectomía (Tabla III y Fig. 3). se supone que esta propuesta permitirá que los cirujanos puedan compartir una descripción común relevante en términos de riesgos perioperatorios y de riesgo de recurrencia de EQ (Vuitton et al., 2020). Para intervenciones no quirúrgicas, se mantienen los acrónimos PAIR (punción, aspiración, inyección, reaspiración, a través de una aguja, sin cateterismo), S-CAT (cateterismo estándar) y MoCAT (cateterismo modificado).

\section{CONCLUSIONES}

En este manuscrito se revisaron los principales cambios en la terminología para EQ. Se ha ilustrado que la adopción del termino echinococcosis para designar la enfermedad humana es un error, que ha sido aceptado, para dar uniformidad de conceptos y evitar seguir produciendo confusión epidemiológica y clínica

ROJAS, C. \& MANTEROLA, C. Echinococcosis: Update on terminologies used from biology to therapeutics. Int. J. Morphol., 39(4):1240-1252, 2021.

SUMMARY: The diagnosis and treatment of echinococcosis requires the participation of different multidisciplinary specialists Therefore, standardizing the terminology used is essential. To harmonize terminology on a sound scientific and linguistic basis, the World Echinococcosis Association generated a formal consensus process. The main achievements of this process were to update the current nomenclature of Echinococcus spp .; to generate an agreement on names of diseases caused by Echinococcus spp .: cystic echinococcosis (CE), alveolar echinococcosis (EA) and neotropical echinococcosis (NE), and the exclusion of other names; execute an agreement on the restricted use of the adjective "hydatid" to refer to the cyst and the fluid due to E. granulosus sensu lato; and an agreement on a standardized description of surgical interventions for $\mathrm{CE}$, according to the framework of "Cyst Access, Opening, Resection and Integrity" (AARI). In addition, 95 "approved" and 60 "rejected" terms were listed. The recommendations provided will be applicable to scientific publications and communication with professionals. The aim of this manuscript was to generate a study document referring to echinococcosis from biology to therapy.

KEY WORDS: Echinococcus; Echinococcosis; Alveolar echinococcosis [Supplementary Concept]; Terminology as Topic; Cystic echinococcosis; Neotropical echinococcosis.

\section{REFERENCIAS BIBLIOGRÁFICAS}

Addy, F.; Wassermann, M.; Banda, F.; Mbaya, H.; Aschenborn, J.; Aschenborn, O.; Koskei, P.; Umhang, G.; DE LA Rue, M.; Elmahdi, I. E.; et al. Genetic polymorphism and population structure of Echinococcus ortleppi. Parasitology, 144(4):450-8, 2017. 
Bittencourt-Oliveira, F.; Teixeira, P.; Alencar, A.; Menezes, R.; Corrêa, C.; Neves, L.; Almeida, F.; Daipert-Garcia, D.; Machado-Silva, J. R. \& Rodrigues-Silva, R. First parasitological, histopathological and molecular characterization of Echinococcus vogeli Rausch and Bernstein, 1972 from Cuniculus paca Linnaeus, 1766 in the Cerrado biome (Mato Grosso do Sul, Brazil). Vet. Parasitol., 250:35-9, 2018.

Booth, M. Climate change and the neglected tropical diseases. Adv. Parasitol., 100:39-126, 2018.

Cordero del Campillo, M. Notas Históricas de la Equinococosis/ Hidatidosis. Inf. Vet., Mayo:23-6, 2008. Disponible en: https:// www.historiaveterinaria.org/update/equinococosis-i-1456736405.pdf

Cornec-Le Gall, E.; Torres, V. E. \& Harris, P. C. Genetic complexity of autosomal dominant polycystic kidney and liver diseases. J. Am. Soc. Nephrol., 29(1):13-23, 2018.

Deplazes, P.; Rinaldi, L.; Alvarez Rojas, C. A.; Torgerson, P. R.; Harandi, M. F.; Romig, T.; Antolova, D.; Schurer, J. M.; Lahmar, S.; Cringoli, G.; et al. Global distribution of alveolar and cystic echinococcosis. Adv. Parasitol., 95:315-493, 2017.

Eckert, J. \& Thompson, R. C. A. Historical aspects of echinococcosis. Adv. Parasitol., 95:1-64, 2017.

Hickman, C.; Roberts, L.; Keen, S.; Larson, A.; L'Anson, H. \& Eisenhour, D. Principios Integrales de Zoología. 16a ed. Madrid, McGraw-Hill/ Interamericana, 2016.

Kassai, T. Nomenclature for parasitic diseases: cohabitation with inconsistency for how long and why? Vet. Parasitol., 138(3-4):169$78,2006 \mathrm{~b}$.

Kassai, T. The impact on database searching arising from inconsistency in the nomenclature of parasitic diseases. Vet. Parasitol., 138(34):358-61, 2006a.

Kassai, T.; Cordero del Campillo, M.; Euzeby, J.; Gaafar, S.; Hiepe, T. \& Himonas, C. A. Standardized nomenclature of animal parasitic diseases (SNOAPAD). Vet. Parasitol., 29(4):299-326, 1988.

Kern, P.; Menezes da Silva, A.; Akhan, O.; Müllhaupt, B.; Vizcaychipi, K. A.; Budke, C. \& Vuitton, D. A. The echinococcoses: diagnosis, clinical management and burden of disease. Adv. Parasitol., 96:259369, 2017.

Kern, P.; Wen, H.; Sato, N.; Vuitton, D. A.; Gruener, B.; Shao, Y.; Delabrousse, E.; Kratzer, W. \& Bresson-Hadni, S. WHO classification of alveolar echinococcosis: principles and application. Parasitol. Int., 55 Suppl::S283-7, 2006.

Macin, S.; Orsten, S.; Samadzade, R.; Colak, B.; Cebeci, H. \& Fındık, D. Human and animal cystic echinococcosis in Konya, Turkey: molecular identification and the first report of $E$. equinus from human host in Turkey. Parasitol. Res., 120(2):563-8, 2021.

Manterola, C.; Benavente, F.; Melo, A.; Vial, M. \& Roa, J. C. Description of Echinococcus granulosus genotypes in human hydatidosis in a region of southern Chile. Parasitol. Int., 57(3):342-6, 2008.

Manterola, C.; Del Sol, M.; Ottone, N. \& Otzen, T. Radiological and surgical anatomy of the liver and fundamentals of the various options liver resections. Int. J. Morphol., 35(4):1517-24, 2017.

Manterola, C.; Rojas, C.; Totomoch-Serra, A.; García-Méndez, N. \& Riffo-Campos, A. L. Echinococcus granulosus genotypes verified in human hydatid disease around the world. Systematic review. Rev. Chil. Infectol., 37(5):541-9, 2020

Manterola, C.; Totomoch-Serra, A.; Rojas, C.; Riffo-Campos, A. L. \& García-Méndez, N. Echinococcus granulosus sensu lato genotypes in different hosts worldwide: a systematic review. Acta Parasitol., 2021. DOI: https://www.doi.org/10.1007/s11686-021-00439-8

Manterola, C.; Urrutia, S. \& Grupo MINCIR. Post surgery morbidity in patients with complicated hepatic hydatidosis. Rev. Chil. Infectol., 32(1):43-9, 2015

Manterola, C.; Vial, M.; Melo, A.; Oberg, C. \& Fonseca, F. Viability and fertility of human hepatic hydatid cysts. World J. Surg., 30(2):22732,2006

McManus, D. P. Current status of the genetics and molecular taxonomy of Echinococcus species. Parasitology, 140(13):1617-23, 2013.
Nakao, M.; Lavikainen, A.; Yanagida, T. \& Ito, A. Phylogenetic systematics of the genus Echinococcus (Cestoda: Taeniidae). Int. J. Parasitol. 43(12-13):1017-29, 2013.

Romig, T.; Deplazes, P.; Jenkins, D.; Giraudoux, P.; Massolo, A.; Craig, P. S.; Wassermann, M.; Takahashi, K. \& de la Rue, M. Ecology and life cycle patterns of echinococcus species. Adv. Parasitol., 95:213-314, 2017.

Tsai, I. J.; Zarowiecki, M.; Holroyd, N.; Garciarrubio, A.; Sánchez-Flores, A.; Brooks, K. L.; Tracey, A.; Bobes, R. J.; Fragoso, G.; Sciutto, E.; et $a l$. The genomes of four tapeworm species reveal adaptations to parasitism. Nature, 496(7443):57-63, 2013.

Turnes, A. La Hidatidosis como Problema de Salud Pública. Una Mirada Histórica. [Internet]. Decima. Montevideo, Sindicato Médico del Uruguay, 2009. Disponible en: https://www.smu.org.uy/dpmc/hmed/historia/articulos/hidatidosis.pdf

Vuitton, D. A.; McManus, D. P.; Rogan, M. T.; Romig, T.; Gottstein, B.; Naidich, A.; Tuxun, T.; Wen, H.; Menezes da Silva, A. \& World Association of Echinococcosis. International consensus on terminology to be used in the field of echinococcoses. Parasite, 27:41, 2020.

Vuitton, D. A.; Wang, Q.; Zhou, H. X.; Raoul, F.; Knapp, J.; Bresson-Hadni, S.; Wen, H. \& Giraudoux, P. A historical view of alveolar echinococcosis, 160 years after the discovery of the first case in humans: part 1. What have we learnt on the distribution of the disease and on its parasitic agent? Chin. Med. J. (Engl)., 124(18):2943-53, 2011.

Wen, H.; Vuitton, L.; Tuxun, T.; Li, J.; Vuitton, D. A.; Zhang, W. \& McManus, D. P. Echinococcosis: advances in the 21st Century. Clin. Microbiol. Rev., 32(2):e00075-18, 2019.

WHO Informal Working Group. International classification of ultrasound images in cystic echinococcosis for application in clinical and field epidemiological settings. Acta Trop., 85(2):253-61, 2003.

World Health Organization. Neglected Zoonotic Tropical Diseases. Ginebra, World Health Organization, 2015. Disponible en: https:// www.who.int/news-room/facts-in-pictures/detail/neglected-zoonotictropical-diseases.

Zhang, W.; Wang, S. \& McManus, D. P. Echinococcus granulosus genomics: a new dawn for improved diagnosis, treatment, and control of echinococcosis. Parasite, 21:66, 2014

Zheng, H.; Zhang, W.; Zhang, L.; Zhang, Z.; Li, J.; Lu, G.; Zhu, Y.; Wang, Y.; Huang, Y.; Liu, J.; et al. The genome of the hydatid tapeworm Echinococcus granulosus. Nat. Genet., 45(10):1168-75, 2013.

\section{Dirección para correspondencia: \\ Dr. Carlos Manterola}

CEMyQ

Universidad de La Frontera

Casilla 54-D

Temuco

CHILE

\section{E- mail: carlos.manterola@ufrontera.cl}

Recibido : 14-03-2021

Aceptado: 26-05-2021 